\title{
Scanning SQUID Microscope Study of Vortex Polygons and Shells in Weak Pinning Disks of an Amorphous Superconducting Film
}

\author{
Nobuhito Kokubo ${ }^{1}$, Satoru Okayasu ${ }^{2}$, Akinobu Kanda ${ }^{3}$, and Bunju Shinozaki ${ }^{4}$ \\ ${ }^{1}$ Center for Research and Advancement in Higher Education, Kyushu University, Fukuoka 819-0395, Japan \\ ${ }^{2}$ Advanced Science Research Center, Japan Atomic Research Institute, Ibaraki 319-1195, Japan \\ 3 Institute of Physics, University of Tsukuba, Tsukuba 305-8571, Japan \\ 4 Department of Physics, Kyushu University, Fukuoka 812-8581, Japan
}

(March 25, 2022)

\begin{abstract}
Direct observation of vortices by the scanning SQUID microscopy was made on large mesoscopic disks of an amorphous MoGe thin film. Owing to the weak pinning nature of the amorphous film, vortices are able to form geometry induced, (quasi-)symmetric configurations of polygons and concentric shells in the large disks. Systematic measurements made on selected disks allow us to trace not only how the vortex pattern evolves with magnetic field, but also how the vortex polygons change in size and rotate with respect to the disk center. The results are in good agreement with theoretical considerations for mesoscopic disks with sufficiently large diameter. A series of vortex images obtained in a disk with a pinning site reveals a unique line symmetry in vortex configurations, resulting in modifications of the shell filling rule and the magic number.
\end{abstract}

PACS numbers: 74.78.Na, 74.78.-w

\section{INTRODUCTION}

Tiny superconductors, accommodating only a few quantized magnetic flux lines, display a great variety of vortex structure different from the Abrikozov vortex lattice in bulk superconductors [1]. Examples include vortex polygons and concentric vortex rings called "vortex shells", which are stable configurations of the repulsive flux lines under the geometrical confinement via the influence of the screening current flowing along the sample edge. The issue of vortex matter confined into small superconductors has been studied theoretically and numerically for many years, focusing mainly on how the vortices are distributed in disk [2] [3] [4] [5] [6] [7], square [8], and triangle shaped small superconductors [9]. Several studies reveal the field evolutions of the vortex structure in small superconductors, and argue which vortex configurations are energetically favorable and how the transition between different vortex states occurs. The obtained rule of shell filling and magic number configurations for consecutive new shells are relevant to phenomena observed in other systems, including the puzzling nucleation of vortices observed in rotating condensates of superfluid ${ }^{4} \mathrm{He}$ [10] and cold dilute alkali-metal gases [11].

The experimental investigations for vortex states in small superconductors were initiated by Hall magnetometer measurements [12] [13], followed by a multiple-small tunneling junction measurement [14]. Kinks in the magnetization or jumps in the tunneling spectra mark transitions of vortex states in small superconducting dots. They evidence changes of vorticity $L$ (the number of vortices), not actual distributions of vortices in the dots.

The visualization of vortices in small superconductors was reported earlier in studies of the scanning superconducting quantum interference device (SQUID) mi- croscopy [15] [16] and later in studies of the scanning Hall probe microscopy [17] [18], showing reasonable images of vortex configurations for $L$ up to 6 . More vortices have been imaged in recent Bitter decoration studies as patterns of magnetic particles deposited over micrometersized dots of $\mathrm{Nb}$ films [19] [20] and Nb mesas [21]. The experiments reveal the rule of the shell filling with magic numbers for $L$ up to 40 by combining vortex configurations visualized over many disks at different magnetic fields, although some features influenced by the bulk pinning and/or the roughness of the disks are involved.

The scanning SQUID microscope technique, which we employed in this study, is an alternative and complement method to visualize vortices in the superconducting dots. By scanning a small pick-up loop over the dots, one can magnetically image the vortices without damaging the samples. This allows us to study systematically how the vortex configuration evolves with magnetic field in a specific sample. While the scanning SQUID microscope has attained the best magnetic sensitivity (better than $5 \mu \Phi_{0} / \sqrt{\mathrm{Hz}}$ ), the spatial resolution is limited typically to a few micrometer due to the size of the pick-up loop $(\sim 10 \mu \mathrm{m})$ [22]. This implies that the observable vortex density is low $(<100 \mu \mathrm{T})$ and the sample becomes much larger than the loop size. The earlier studies were made on YBCO [15] and $\mathrm{Nb}$ dots [16] with $\sim 50 \mu \mathrm{m}$ in size, which is decades larger than the penetration depth $\lambda$ or the coherence length $\xi$ of the superconducting materials. In such large dots, the effect of the geometrical confinement is weak and (sparse) vortices form likely disordered configurations due to the dominant influence of the bulk pinning in the superconducting materials [15] [16].

If the influence of the bulk pinning is reduced by using weak pinning, superconducting materials, the situation can be different: the geometrical confinement dominates 
over the bulk pinning. Moreover, when the dots become thin in thickness $d(<2 \lambda)$, the interaction between vortices becomes long ranged and it decays slowly with the vortex-vortex spacing $r$ as $\propto 1 / r$ for $r>2 \lambda^{2} / d$ [23], in contrast to the short ranged interaction characterized by the exponential decay $\propto e^{-r / \lambda}$ for $r>\lambda$ in the bulk superconductors. Thus, even in the large dots the interplay between the vortex-vortex interaction and the geometrical confinement may lead to geometry-induced, symmetric configurations of the sparse vortices in the presence of the weak bulk pinning. Here, we report on the direct observation of vortices in large thin disks of an amorphous $(\alpha)$ MoGe film by the scanning SQUID microscopy. Due to the weak bulk pinning of the amorphous film, we are able to observe (quasi-) symmetric configurations of polygons and concentric shells of vortices for $L$ up to 19. The results illustrate not only how the vortex configuration evolves with the magnetic field, but also how the vortex polygons change in size and rotate with respect to the disk center. We present vortex images obtained in a disk with a pinning site and discuss how vortex configurations are altered by a pinned vortex.

This paper is outlined as follows: After describing experimental details in Sec. II, we present vortex polygon states observed for small vorticities up to $L=5$ in Sec. III. In Sec. IV we present field evolutions of vortex shells observed for large vorticities $L \geq 6$. The influence of a pinning site on vortex configurations is discussed in Sec. V. In Sec. VI comparison with other experiment is made. Our summary is given in Sec. VII.

\section{EXPERIMENTAL}

We used a commercial scanning SQUID microscope (SQM-2000, SII Nanotechnology) with a dc SQUID magnetometer made of $\mathrm{Nb} / \mathrm{Al}-\mathrm{AlO}_{x} / \mathrm{Nb}$ Josephson junctions and an inductively coupled, pick-up loop of a $\mathrm{Nb}$ film [16]. The pick-up loop had $10 \mu \mathrm{m}$ in diameter with $2 \mu \mathrm{m}$ line width. The dc SQUID and the pick-up loop were integrated on a square Si chip of $3 \times 3 \mathrm{~mm}^{2}$ in size, which was mounted on a phosphor bronze cantilever. The sensor chip was tilted with respect to the sample stage by a shallow angle of $\sim 10$ degrees and a corner of the chip was softly in contact with the sample surface, in order to keep a short distance ( $\sim 5 \mu \mathrm{m}$ at the minimum) between the loop and the sample surface. This ensures safe operation of the scanning SQUID microscope with reasonably high spatial resolutions $(\sim 4 \mu \mathrm{m})$. The whole assembly including the sensor chip and the sample stage was surround by a $\mu$-metal shield, resulting in a residual magnetic field (ambient field) of $\sim 1 \mu \mathrm{T}$. A coil wound in our sample stage allowed us to apply small magnetic field $H$ perpendicular to the sample. We scanned the sample stage in XY directions by dc stepper motors. In order to minimize the possible mechanical hysteresis effect, we took one scan direction from the left side to the right side of the image. The scanning speed was about 5 pixels $/ \mathrm{s}$ and this resulted in an image of $64 \times 64$ pixels by typically 15 minutes. The temperature for the SQUID sensor was $2-3 \mathrm{~K}$, while that for the sample stage was varied.

We prepared weak pinning disks of an $\alpha-\mathrm{Mo}_{x} \mathrm{Ge}_{1-x}$ $(x \approx 78 \%)$ thin film with a lithographic technique. After preparing a silicon substrate covered with a patternedresist film, we sputtered the $\alpha$-MoGe film with $0.22 \mu \mathrm{m}$ thickness on top. Employing the lift-off technique, we patterned the film into arrays of disks with four different diameters $D_{d}$ of $20 \mu \mathrm{m}, 34 \mu \mathrm{m}, 56 \mu \mathrm{m}$ and $106 \mu \mathrm{m}$. The spacing between the disks was chosen to be large enough ( $>200 \mu \mathrm{m})$, so that the interdisk coupling can be excluded. In order to reduce possible damage during the scanning, all the disks were covered with $0.1 \mu \mathrm{m}$ thick $\mathrm{SiO}_{2}$ film as a protective layer. The material parameters relevant to this study are as follows: the superconducting transition temperature $T_{c}$ is $6.0 \mathrm{~K}$, the coherence length $\xi(0)$ at $T=0$ is $4.5 \mathrm{~nm}$, and the penetration depth $\lambda(0)$ at $T=0$ is $0.58 \mu \mathrm{m}(\gg \xi(0))$ [24]. Since the thickness is smaller than $\lambda(0)$, the penetration depth is expanded to the effective one given by $\Lambda_{e f f}(0)=2 \lambda^{2}(0) / d(\gg \lambda(0))$. For the present film $\Lambda_{\text {eff }}(0) \sim 3.1 \mu \mathrm{m}$. Normalizing the disk size by the effective penetration depth, we find $D_{d} \approx$ 6-35 $\Lambda_{\text {eff }}(0)$, indicating large mesoscopic disks.

In weak pinning materials, the distance $z_{0}$ between the pick-up loop and the sample surface is an important parameter to be controlled in order to obtain clear images of vortices. As demonstrated by Plourde et al. [25], the vortices may be dragged and/or shifted in positions by the pick-up loop during the scanning. This coupled motion of vortices can be reduced when $z_{0}$ is increased by lowering the sample stage from the contact point with the sensor chip. This procedure, however, results in a smeared image of vortices due to the spread of the magnetic flux above the sample surface [26]. We could not resolve individual vortices when $z_{0}$ became more than the loop diameter $(>10 \mu \mathrm{m})$. Hence, we set $z_{0} \sim 7 \mu \mathrm{m}$ as a typical distance. All the images presented in this study were taken in the field-cool measurement, where the magnetic field was applied in some temperatures $(\sim 10 \mathrm{~K})$ above $T_{c}$, followed by cooling the samples in the magnetic field to temperatures $(\sim 3-5 \mathrm{~K})$ below $T_{c}$ where scanning SQUID measurements were done. This procedure results in the equilibrium distribution of vortices. Because of the possible motion of vortices during the scanning, each image was taken in a single scan made on a fresh vortex state prepared by the field-cool procedure. No image taken by repeated scans is presented.

\section{VORTEX CONFIGURATIONS FOR SMALL VORTICITIES}

First we focus on vortex configurations observed for small vorticities up to 5. Figs. 1(a)-1(f) show selected vortex images observed in a $34 \mu \mathrm{m}$ disk. A color bar 
indicates the magnitude of the magnetic flux in the pickup loop. These images display clearly individual vortices with reasonable spatial and magnetic resolutions [27]. The imaged vortex size is larger than $2 \Lambda_{e f f}(\sim 7$ $\mu \mathrm{m})$ due to defocusing effect originating from the large distance $z_{0}(\sim 7 \mu \mathrm{m})$ between the loop and the sample surface. Details of the field profile of vortices have been reported in Refs. [23] [28] [29]. We find that the vortices form symmetric configurations with respect to the disk center. The vortex pattern evolves with field as follows; After the Meissner state where the magnetic field is expelled from the disk, a single vortex appears in the disk and it sits at the disk center (Fig. 1(a)). Then, two vortices are located symmetrically with respect to the disk center (Fig. 1(b)), followed by the formation of a triangle (Fig. 1(c)), a square (Fig. 1(d)) and a pentagon (Fig. $1(\mathrm{e}))$ of vortices in the disk. Although some distortion is visible for the square, these symmetric configurations are nearly identical to those reported in the Bitter decoration [19] and the theoretical studies [5] [6] [7].
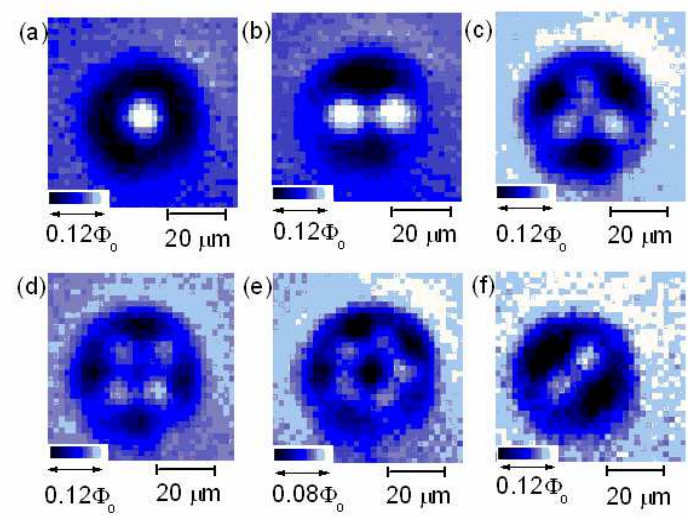

FIG. 1. Scanning SQUID microscope images of vortices in an amorphous MoGe disk with $34 \mu \mathrm{m}$ diameter for vorticities $L=1-5$ obtained after the disk was cooled to $3.2 \mathrm{~K}$ in different magnetic fields. (a) $L=1$ at $8.0 \mu \mathrm{T}$; (b) $L=2$ at $10 \mu \mathrm{T}$; (c) $L=3$ at $16 \mu \mathrm{T}$; (d) $L=4$ at $18 \mu \mathrm{T}$; (e) $L=5$ at $21 \mu \mathrm{T}$. (f) An additional image of the vortex pair at $14 \mu \mathrm{T}$. All images are the same in size of $66^{*} 66 \mu \mathrm{m}^{2}$. Color bars indicate the magnitude of the magnetic flux detected in the pick-up loop.

An important remark for the observation is that some of the vortex patterns rotate with respect to the disk center. This feature is clearly visible in the images of the vortex pair shown in Figs. 1 (b) and 1(f). In Fig. $1(\mathrm{~b})$, the vortex pair is aligned nearly with the horizontal direction of the image, while in Fig. 1 (f) it rotates by $\sim 45^{\circ}$ with respect to the disk center. Other orientation of the pair is observed in other scan, indicating the rotational degree of freedom in the vortex configuration [30]. Similar rotation is observed for the triangle pattern, but not clearly for the square and the pentagon patterns.

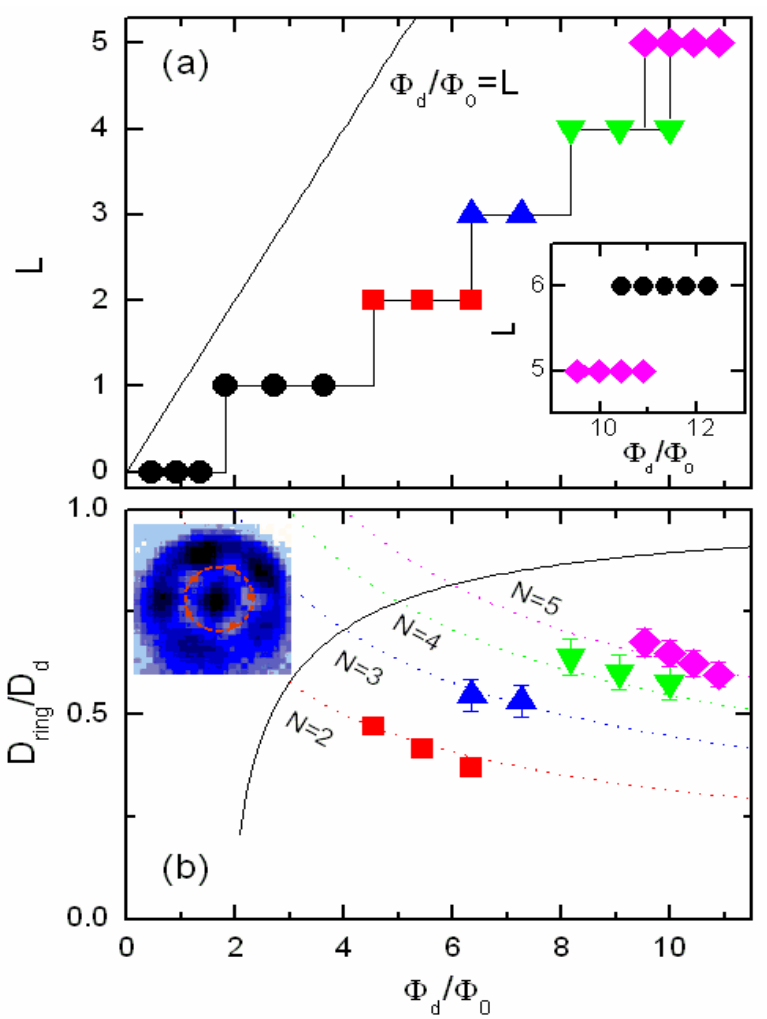

FIG. 2. (a) Vorticity vs. normalized magnetic flux in the $34 \mu \mathrm{m}$ disk in $3.2 \mathrm{~K}$. A solid line represents a condition of $\Phi_{d} / \Phi_{0}=L$. The inset to (a) shows the results around the transition between $L=5$ and 6 obtained by repeated field-cool measurements. (b) The normalized diameter of the polygon ring vs. the normalized magnetic flux. The dotted curves represent the theoretical results for polygon sizes with different $N=2-5$ as denoted. The solid curve indicates the upper limit of the polygon size. The inset to (b) illustrates how we determine the diameter $D_{\text {ring }}$ of the polygon ring.

Next we show how the number of vortices evolves with applied magnetic field. Counting the number of vortices in the images taken at various magnetic fields, we plot the results in Fig. 2(a). Here, instead of the field strength $H$, we use the magnetic flux $\Phi_{d}\left(=\mu_{0} H \pi\left(D_{d} / 2\right)^{2}\right)$ in the disk normalized by the flux quantum $\Phi_{0}$. As observed, the vorticity $L$ shows a stair case behavior with field: After the Meissner state $(L=0)$, the single vortex state $(L=1)$ appears at $\Phi_{d} / \Phi_{0} \approx 2$, and it continues until $\Phi_{d} / \Phi_{0} \approx 4.5$, above which the vortex pair state $(L=$ $2)$ appears. The vortex triangle $(L=3)$, square $(L=$ $4)$ and pentagon $(L=5)$ states appear at $\Phi_{d} / \Phi_{0} \approx 6.4$, 8.2 and 9.5 , respectively. We note that the stair case behavior is well below the condition of $\Phi_{d} / \Phi_{0}=L$ where the magnetic field penetrates fully the disk and this is represented by a solid line. The difference from the line becomes large on increasing field. At $\Phi_{d} / \Phi_{0}=8$, for instance, $\Phi_{d} / \Phi_{0}-L=4$, which is half of $\Phi_{d} / \Phi_{0}$. This indicates a large diamagnetic response of the disk and it implies the presence of the large screening current near 
the disk edge.

The influence of the screening current upon vortex configurations is found by considering how the size of vortex polygons changes with magnetic field. As exemplified in the inset to Fig. 2(b), we draw a circle circumscribing the positions of vortices and estimate the diameter $D_{\text {ring }}$ of the polygon ring. The results for this analysis made at different fields and different vorticities (down to $L=2$ ) are summarized in the main panel of Fig. 2(b). As observed, $D_{\text {ring }}$ varies largely with $L$. For example, $D_{\text {ring }}$ at $L=2$ is roughly $40 \%$ of the disk diameter, while that at $L=5$ becomes expanded to roughly $65 \%$ of the disk diameter. Focusing on the results at each vorticity, we find $D_{\text {ring }}$ decreases with increasing field. The shrinkage of $D_{\text {ring }}$ is observed to be $5-10 \%$ of the disk diameter, depending on $L$.

The obtained results are in excellent agreement with the theoretical considerations for vortex polygons confined by the screening current near the disk edge [2] [6]. In the London limit, the diameter of a vortex polygon at a given magnetic field is written as

$$
D_{\text {ring }}=D_{d} \sqrt{\frac{N-1}{\Phi_{d} / \Phi_{0}}},
$$

where $N$ is the number of vortices on the polygon ring. The behaviors for $N=2-5$ are represented by dotted curves in Fig. 2(b). Our results for $L=N=2-5$ follow nicely those curves without any adjustable parameters. Moreover, they are satisfactory for the condition that the polygon size should be below the maximum diameter above which the formation of the polygon ring is not stable. This is represented by a solid curve and it follows the condition [6] given by

$$
D_{\text {ring }}=D_{d} \sqrt{1-\frac{2}{\Phi_{d} / \Phi_{0}}} .
$$

Thus, we are convinced that the vortex configurations observed in the $34 \mu \mathrm{m}$ disk are dominantly influenced by the geometrical confinement via the screening current near the disk edge. We note, to the best of our knowledge, that these quantitative agreements, together with the rotation of the vortex patterns in the same disk, are not reported in other experimental studies so far.

\section{VORTEX CONFIGURATIONS FOR LARGE VORTICITIES}

For vorticities more than 6 , we observe the formation of concentric shell rings of vortices. Since the vortices become dense and their magnetic images are overlapped each other, for clarity, we present here the results obtained in larger disks. Figs. 3(a)-3(c) show vortex images for $L=6-8$ observed in a disk with $56 \mu \mathrm{m}$ diameter. One can clearly see the shell formation of vortices. In the image of Fig. 3(a), for example, a vortex sits at the center of the disk, while the others form a ring around. Using the standard notation, this configuration is referred to as $(1,5)$, meaning that the inner shell has one vortex and the outer shell has five vortices. After the $(1,5)$ configuration, we observe $(1,6)$ and $(1,7)$ configurations, which are shown in Figs. 3(b) and 3(c), respectively. Namely, the increase of vorticity in the disk results in the growth of the outer shell ring.
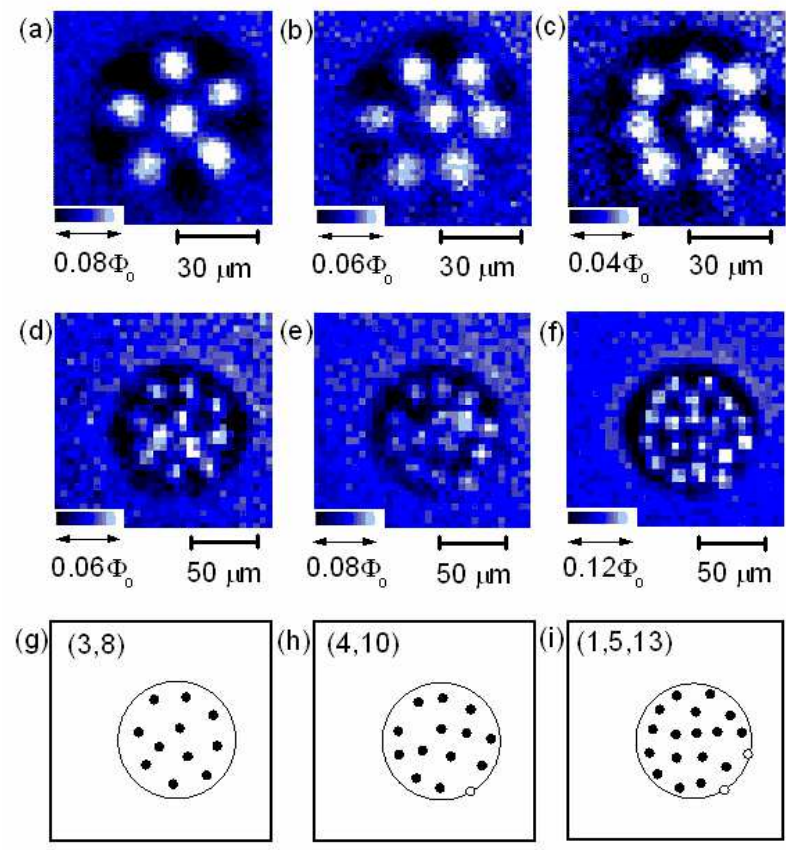

FIG. 3. Images of vortices in disks with different diameters for large vorticities. Images of (a), (b) and (c) are obtained in a $56 \mu \mathrm{m}$ disk in $3.9 \mathrm{~K}$ at fields of $8.0 \mu \mathrm{T}, 9.0 \mu \mathrm{T}$, and $10 \mu \mathrm{T}$, respectively. Other images of (d), (e), and (f) are observed in a $106 \mu \mathrm{m}$ disk in $5.0 \mathrm{~K}$ at fields of $3.8 \mu \mathrm{T}, 4.6 \mu \mathrm{T}$, and 6.0 $\mu \mathrm{T}$, respectively. For clarity, the corresponding vortex configurations for these images of (d), (e), and (f) are sketched in (g), (h), and (i), respectively.

The growth of the inner shell occurs at even larger $L$. Fig. 4 shows the field evolution of vorticity, together with vortex configuration, observed in the largest disk with $106 \mu \mathrm{m}$ diameter. The vortex shells with a single vortex at the center are observed for $L=6,7$, and 8 . This is consistent with the observation made on the 56 $\mu \mathrm{m}$ disk discussed above. At $L=9$ a vortex pair appears as the inner shell, while the others form the outer shell ring around, resulting in a $(2,7)$ configuration. The pair configuration of the inner shell is also observed at $L=10$. At $L=11$ three vortices form a triangle as the inner shell, resulting in a $(3,8)$ configuration. The triangular inner shell is observed over some field range and persists up to $L=13$. In contrast, the appearance of 4 vortices as the inner shell occurs only at $L=14$. At $L=15$ five vortices form a pentagon as the inner shell. The vortex shells with the pentagon inner ring appear in vorticities up to 
$L=17$ above which three concentric shells are formed.

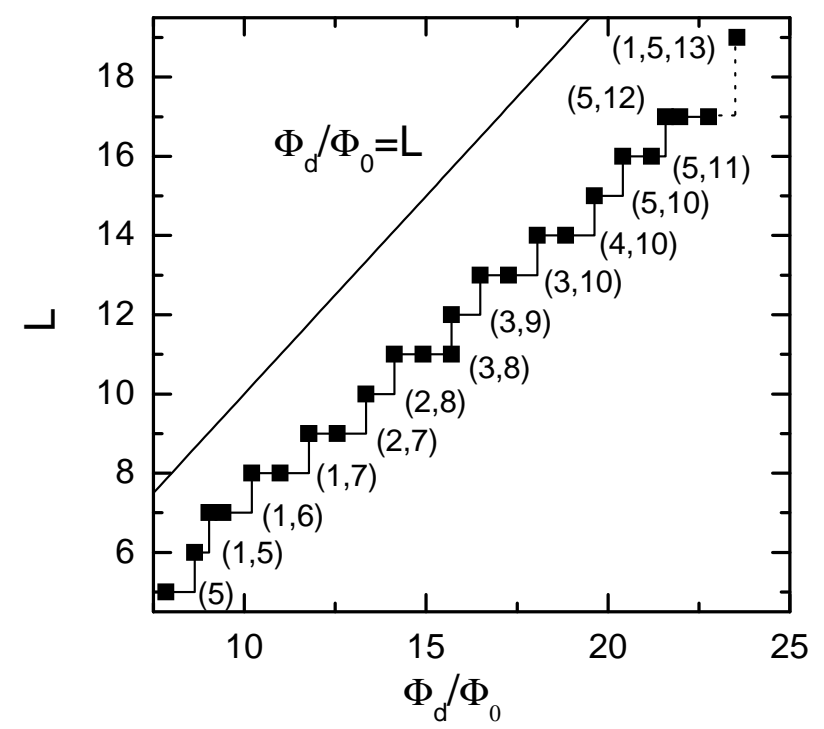

FIG. 4. Vorticity vs. normalized magnetic flux in the 106 $\mu \mathrm{m}$ disk in $5.0 \mathrm{~K}$. The configurations of vortices are denoted. A solid line represents a condition of $\Phi_{d} / \Phi_{0}=L$.

These results follow mostly the rule of the shell filling obtained in theoretical and numerical studies [5] [7]. This includes the magic number configuration at $L=6$ where the first formation of two concentric shells occurs, and also the formation of $(2,7)$ at $L=9$ where two configurations of $(1,8)$ and $(2,7)$ are energetically comparable but the latter one becomes the ground state for the large disk [7]. However, some differences are found for large vorticities $(\geq 13)$. The two shell configurations with 4 vortices in the inner ring are shown to appear for $13 \leq L \leq 15$, while we observe only the $(4,10)$ configuration at $L=14$. Moreover, after the pentagon inner shell at $L=16$ the formation of three vortex shells is shown to occur at $L=$ 17, which is not observed above.

A physical origin for the differences would be found in actual vortex configurations observed in the largest disk. Selected images are shown in Figs. 3(d)-3(f). For clarity, the corresponding vortex configurations are sketched in Figs. 3(g)-3(i). Although some distortions are present, one can reasonably find quasi-symmetric shell formation of vortices even in the largest disk. This is exemplified by Figs. 3(d) and 3(g), where three vortices form a triangle in the inner shell surrounded by 8 vortices as the outer shell ring around. Focusing on vortex configurations in the inner shell, we find the formation of a pair, the triangle and a pentagon, of which patterns are similar to those observed for small vorticities $(L \leq 5)$ given in Fig. 1. However, this is not the case for a square. As shown in Figs. 3(e) and 3(h), the vortices in the inner shell form a strongly deformed square like a rhombus. As discussed below, this originates from the effect of the bulk pinning quenched on the $\alpha$-MoGe film.

We often observe a vortex (or vortices) close to the rim of the disk for large vorticities, which could be attributed to possible roughness of the disk boundary. This is visible in the configurations of $(4,10)$ (Figs. 3(e) and $3(\mathrm{~h})$ ) and $(1,5,13)$ (Figs. 3(f) and 3(i)). As denoted by an open circle $(\mathrm{s})$ in the corresponding sketch, a vortex (vortices) appears close to the lower-right rim of the disk. More rim vortices are occasionally observed when the field-cool procedure is repeated in the same magnetic field, indicating extra vortices trapped in the rim during the field-cool process. If we neglect the rim vortex (vortices), the differences between the experimental observation and the theoretical prediction in vortex configurations found for $L \geq 13$ would be partly explained. In Fig. $3(\mathrm{~h})$, the rim vortex becomes a part of the outer shell ring. If we neglect the vortex, the configuration becomes $(4,9)$. This is consistent with the first configuration for 4 vortices in the inner shell given theoretically [5] [7]. Similarity is applicable to the $(1,5,13)$ configuration shown in Fig. $3(\mathrm{i})$, where two rim vortices are recognized. Thus, the configuration could be reduced to $(1,5,11)$, which is consistent with the magic number configuration for three vortex shells [5] [7]. Further studies would be needed to clarify the origin and the role of the rim vortices on the vortex configurations in details.

Repeating field-cool measurements over some field range, we observe configurations with different vorticities at the same magnetic fields [31]. An example obtained on the $34 \mu \mathrm{m}$ disk is given to the inset to Fig. 2(a). Although we take the same procedure of the field cooling, both the $L=5$ polygon and $L=(1,5)$ shell are observed at the fields of $\Phi_{d} / \Phi_{0}=10.4$ and 10.9 , resulting in some field crossover in the transition between the $L=5$ and $L=(1,5)$ states. Similar crossover may be present in every step in the stair-case behavior of $L$ given to Fig. 2(a) and Fig. 4.

\section{INFLUENCE OF A PINNING SITE UPON VORTEX CONFIGURATIONS}

Out of over 20 disks measured in this study, we found that some disks have a relatively strong pinning site(s) [32], although no defect is visible under an optical microscope. The pinning center(s) is probably introduced during the sample preparation and it originates from the slight compositional inhomogeneity or subtle roughness in the disk. The influence of the pinning site(s) on vortex configurations depends highly on where it is present in the disk. As an example, we present the results (for $L$ up to 13) obtained on a $56 \mu \mathrm{m}$ disk with a pinning site near the disk center.

Figs. 5(a)-5(i) show selected vortex images observed in the disk. We find the pinning site from the image at $L=$ 1 shown in Fig. 5(a), where a vortex is unnaturally offcentered in the disk [33]. This is against the geometrical confinement and it should be attributed to the influence of the pinning center. Thus, we identify the vortex posi- 
tion as the pinning site and mark a red dot on the image. No more pinning center is found in the disk.

The rest of the images indicate clearly how other vortices are distributed in the presence of the vortex trapped at the pinning site as marked by the red dot. Fig. 5(b) shows the image of two vortices in the disk: One of them sits at the pinning site, while the other appears in the opposite side with respect to the disk center. We do not observe any orientational changes in the pair even when the magnetic field is varied. Similarity is applicable to the image of a triangle configuration given to Fig. 5 (c). Thus, the influence of the pinning site fixes the vortex patterns and it breaks the rotational degree of freedom in the vortex configurations.

The deformation of vortex polygons occurs for vorticities more than 4 . Fig. 5(d) shows the image of 4 vortices in the disk. The vortices form a rhombus-shaped configuration, where the diagonal distance between the pinned vortex and the diagonal vortex is shorter than that for the other two vortices. This is similar in shape to the disordered square in the inner shell observed in the largest disk (see Figs. 3(e) and 3(h)).

A unique configuration of vortices is observed in the image at $L=6$. As shown in Fig. 5(f), one can see two rows of three vortices like a dice pattern of six with the pinned vortex in the middle of the upper row. This pattern is stable against some field change and no shell pattern is observed at $L=6$. A precursor of the formation of the rows can be seen in the image at $L=5$ (Fig. $5(\mathrm{e})$ ), where the vortices form a pentagon with deformation inward by the pinned vortex (the top of the pentagon) and higher right three vortices try to be aligned. The formation of the (upper) row remains in the image at $L=7$ (Fig. 5(g)). Thus, the pinning site near the disk center triggers off the formation of the vortex row and stabilizes the dice like vortex pattern at $L=6$.

Because of the dice pattern of vortices at $L=6$, the first formation of two concentric shells occurs at $L=7$. This is larger than $L=6$ where the magic number configuration for two concentric shells is observed in the "pin-free" disks. Thus, the shell filling rule is altered by the pinning site near the disk center.

For $L>7$, the vortex shells are observed with the pinned vortex being a part of the inner shell ring. As in the image of Fig. 5(h), the pinned vortex is involved in the formation of a pair as the inner shell, while the other vortices form the outer vortex ring around. The corresponding sketch given in Fig. 5(k) allows us to identify the configuration as $(2,6)$. To our knowledge, this configuration has not been reported in other studies so far. For larger vorticities, we observe two shell configurations of $(2,7),(2,8)$ and $(3,8)$ which are similar to those observed in the largest disk.
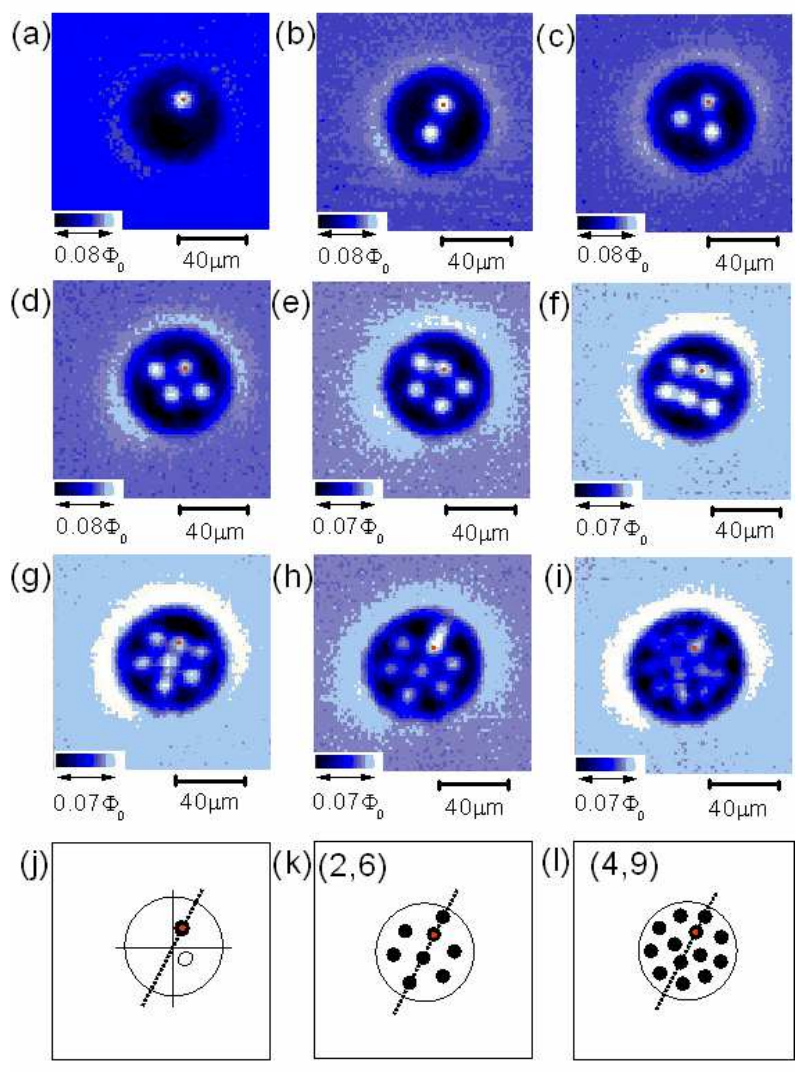

FIG. 5. Selected images of vortices observed in a $56 \mu \mathrm{m}$ disk with a pinning center taken in $3.3 \mathrm{~K}$ for different vorticities. (a) $L=1$ at $4.0 \mu \mathrm{T}$; (b) $L=2$ at $6.0 \mu \mathrm{T}$; (c) $L=3$ at $7.5 \mu \mathrm{T}$; (d) $L=4$ at $9.0 \mu \mathrm{T}$; (e) $L=5$ at $10 \mu \mathrm{T}$; (f) $L=6$ at $11 \mu \mathrm{T}$; (g) $L=7$ at $13 \mu \mathrm{T}$; (h) $L=8$ at $13.5 \mu \mathrm{T}$; (i) $L=13$ at $20 \mu \mathrm{T}$. A red point in each image marks the position of the pinning site in the disk. For clarity, the vortex configurations for the images of (a), (h), and (i) are sketched in (j), (k), and (l), respectively. The dotted line for each sketch represents a symmetric line defined by the pinning site and the disk center O.

It is interesting to show the image at $L=13$ given in Fig. 5(i), where 4 vortices form the inner shell surrounded by 9 vortices as the outer shell. As sketched in Fig. 5(l), the vortices in the inner shell form a rhombusshaped, deformed square, while the outer shell vortices form a ring around. Again, focusing on the inner shell, we find that the deformation from the regular square is weaker than that observed at $L=4$ (Fig. 5(d)) and the effect of the pinning site is opposite; Namely, the pinned vortex and the diagonal vortex gives the longer diagonal of the rhombus. Thus, the vortex square is stretched by the influence of the pinning site. We note that this effect depends highly on where the pinning site is present and how large the square polygon is. The regular square may appear if the pinning site is located on the circumference of the ring defined by the regular square vortices.

All the vortex configurations observed in the disk can be characterized by a line symmetry, defined by the 
pinned vortex and the disk center $\mathbf{O}$, as sketched in Fig. $5(\mathrm{j})$. Drawing the line in the sketch of Fig. 5(l), for instance, one can find that the vortex configuration is divided into halves that are nearly the mirror configurations of each other. Similarity is applicable to the other configurations including unique ones at $L=6$ and 8 , although slight deviation is present at $L=5$ and 7 . Note that the odd (even) number of vortices lies on the line when $L$ is odd (even). In the "pin free" disks no unique line symmetry characterizing all the vortex patterns with different vorticities appears. Thus, we are convinced that the line symmetry in the vortex configurations is induced by the off-centered pinning site in the disk. A physical reason for the symmetry is not simple since the vortex patterns are determined by subtle balance of many competing interactions, including the interaction with the bulk pinning, the repulsive interaction between vortices, and the interaction with the disk boundary. To clarify this issue, a numerical simulation study would be needed.

Let us estimate roughly the pinning force $f_{p}$ for the vortex at the pinning site. We find that at $4.5 \mu \mathrm{T}$, just below the step field between $L=1$ and $L=2$ states, the disk has only a single vortex, but it appears at the disk center. This is distinct from the observation made at lower fields of $3.5 \mu \mathrm{T}$ and $4.0 \mu \mathrm{T}$, where the vortex appears at the off-centered pinning site (Fig. 5(a)). These facts indicate the important influence of the force on the vortex due to the geometrical confinement, and suggest that this force should be comparable to the pinning force in between $4.0 \mu \mathrm{T}$ and $4.5 \mu \mathrm{T}$. A model described in Refs. [2], [5] and [21] shows that the force $f^{s}$ of vortex interactions with the shielding current and edge for a given magnetic field is written as

$$
f^{s}=f_{0}\left[\frac{1}{1-\left(\frac{r}{D_{d} / 2}\right)^{2}}-\frac{\Phi_{d}}{\Phi_{0}}\right] \cdot \frac{r}{D_{d} / 2}
$$

where $r$ is the distance of the vortex position from the disk center and $f_{0}\left(=\Phi_{0}^{2} / \pi \mu_{0} D_{d} \lambda^{2} \approx 5.0 \times 10^{-8} \mathrm{~N} / \mathrm{m}\right)$ is the unit of the force for a vortex per unit length. Using the distance $(r=12 \mu \mathrm{m})$ for the off-centered vortex, we find the pinning force as $f_{p} \approx f^{s} \approx 1.8 f_{0}$. This result, together with our observation where the pinning site accommodates only one vortex, are consistent with the numerical simulation made at the weak pinning force of $f_{p} / f_{0}=2[21]$.

Further measurements are made on other disks with a pinning site near the disk edge. It turns out that the pinned vortex near the disk edge gives only some distortion on the vortex $\operatorname{ring}(\mathrm{s})$. No unique configuration is observed except for a highly off-centered vortex configuration at $L=1$. These facts indicate that the pinning site near the disk center provides more strong influence upon the vortex configurations than that near the disk edge.

\section{COMPARISON WITH OTHER EXPERIMENT}

Comparing the results in the present experiment to those found in the Bitter decoration experiment [19], we find that both experiments share the general features of vortex configurations including the shell filling rule and the magic number, although magnetic fields and disk sizes are different in decades. Let us shortly discuss the experimental conditions made on these studies. In the decoration experiment the symmetric vortex shells were observed in smaller disks with 1-5 $\mu \mathrm{m}$ diameter at higher magnetic fields $(\sim \mathrm{mT})$. Due to the small disk size, the geometrical confinement is strong and it dominates over the influence of the strong bulk pinning in the $\mathrm{Nb}$ film. Meanwhile, in the present experiment, the disk size is $\sim 10$ times larger and the field range is two decades smaller. The corresponding average vortex spacing is $\sim 10 \mu \mathrm{m}$, which is larger than the effective penetration depth. Thus, the interaction between vortices as well as the influence of the edge current are weaker, but they remain effective in the vortex configurations because the bulk pinning in the amorphous film is weak. This situation is validated in the observation of a reasonably ordered configuration of vortices in $\alpha$-MoGe thin films at a low field of $10 \mu \mathrm{T}$ [29]. Moreover, normalizing the disk size by the effective penetration depth, we find that our disk size $(6-35 \Lambda(0))$ is not much different from that of 9-46 $\Lambda(0)$ for the decoration experiment. These facts not only link two experiments made in different sizes and different field ranges, but also confirm that our observation is made in the large mesoscopic regime.

\section{SUMMARY}

We presented the scanning SQUID microscope images of vortices observed in the weak pinning, $\alpha$-MoGe thin disks with different diameters. The observed images illustrate clearly geometry-induced, (quasi-)symmetric configurations of vortices for $L$ up to 19 .

For small vorticities up to 5 , the vortices form the symmetric polygons with respect to the disk center. The polygon size grows with $L$, while it shrinks by the enhancement of the screening current. Some of the polygons rotate clearly with respect to the disk center, indicating the rotational degree of freedom in the vortex configurations. The influence of the geometrical confinement is evident in the quantitative agreements between the field evolution of the polygon size and the theoretical considerations in the London limit.

For large vorticities more than 6 , the vortices form the concentric shell rings. The field evolutions of the inner and outer shell rings follow the rule of the shell filling obtained in the theoretical and numerical studies. Some differences are found in vortex configurations for large vorticities $(\geq 13)$ observed in the largest disk, and they 
could be explained partly by the influence of the weak bulk pinning and/or the edge roughness in the disk.

We also presented the vortex images obtained in the disk with the off-centered pinning site. The results reveal the pinning induced, novel configurations of vortices: In addition to the deformed polygons, we observed unique vortex configurations of the dice pattern at $L=6$ and $(2,6)$ at $L=8$. These lead to the pinning induced changes in the shell filling rule and the magic number. All the configurations observed in the disk are nearly symmetric with respect to the line defined by the pinned vortex and the disk center.

\section{ACKNOWLEDGEMENTS}

N. K. thanks O. Narikiyo, H. Kawai, K. Kadowaki, B. J. Baelus, and V. R. Misko for useful discussion. This work was supported partly by the grant in Aid for Scientific research from MEXT (the Ministry of Education, Culture, Sports, Science and Technology), Japan.

Corresponding author: N. Kokubo, Center for Research and Advancement in Higher Education, Kyushu University, 4-2-1, Moto-oka, Nishi-ku, Fukuoka, Fukuoka 819-0395, Japan; e-mail:kokubo(at)rche.kyushu-u.ac.jp.

[1] A. A. Abrikosov, Sov. Phys. JETP 5, 1174 (1957).

[2] A.I. Buzdin and J.P. Brison, Phys. Lett. A 196, 267 (1994).

[3] P. A. Venegas and E. Sardella, Phys. Rev. B 58, 5789 (1998).

[4] J. J. Palacios, Phys. Rev. Lett. 84, 1796 (2000).

[5] B. J. Baelus, L. R. E. Cabral, F. M. Peeters, Phys. Rev. B 69, 064506 (2004).

[6] L. R. E. Cabral, B. J. Baelus, and F. M. Peeters, Phys. Rev. B 70, 144523 (2004).

[7] V. R. Misko, B. Xu, and F. M. Peeters, Phys. Rev. B 76, 024516 (2007).

[8] L. F. Chibotaru, A. Ceulemans, V. Bruyndoncx, V. V. Moshchalkov, Nature 408, 833 (2000).

[9] L. F. Chibotaru, A. Ceulemans, V. Bruyndoncx, and V. V. Moshchalkov, Phys. Rev. Lett. 86, 1323 (2001).

[10] E. Y. Yarmchuck and R. E. Packard, J. Low Temp. Phys. 46, 479 (1982).

[11] K. W. Madison, F. Chevy, W. Wohlleben, and J. Dalibard, Phys. Rev. Lett. 84, 806 (2000).

[12] A. K. Geim, I. V. Grigorieva, S. V. Dubonos, J. G. S. Lok, J. C. Maan, A. E. Filippo, F. M. Peeters, Nature 390, 259 (1997).

[13] A. K. Geim, S. V. Dubonos, J. J. Palacios, I. V. Grigorieva, M. Henini, J. J. Schermer, Phys. Rev. Lett. 85, 1528 (2000).
[14] A. Kanda, B. J. Baelus, F. M. Peeters, K. Kadowaki, Y. Ootuka, Phys. Rev. Lett. 93, 257002 (2004).

[15] Y. Hata, J. Suzuki, I. Kakeya, K. Kadowaki, A. Odawara, A. Nagata, S. Nakayama and K. Chinone, Physica C 388389, 719 (2003).

[16] T. Nishio, S. Okayasu, J. Suzuki, K. Kadowaki, Physica C 412-414, 379 (2004).

[17] T. Nishio, Q. Chen, W. Gillijns, K. De Keyser, K. Vervaeke, and V. V. Moshchalkov, Phys. Rev. B 77, 012502 (2008).

[18] M. R. Connolly, M. V. Milosevic, S. J. Bending, J. R. Clem and T. Tamegai, Euro Phys. Lett. 85, 17008 (2009).

[19] I. V. Grigorieva, W. Escoffier, J. Richardson, L. Y. Vinnikov, S. Dubonos, V. Oboznov, Phys. Rev. Lett. 96, 077005 (2006).

[20] H. J. Zhao, V. R. Misko, F. M. Peeters, V. Oboznov, S. V. Dubonos and I.V. Grigorieva, Phys. Rev. B 78, 104517 (2008).

[21] I.V. Grigorieva, W. Escoffier, V. R. Misko, B. J. Baelus, F. M. Peeters, L. Y. Vinnikov, and S. V. Dubonos, Phys. Rev. Lett. 99, 147003 (2007).

[22] J. R. Kirtley, M. B. Ketchen, K. G. Stawiasz, J. Z. Sun, W. J. Gallagher, S. H. Blanton, and S. J. Wind Appl. Phys. Lett. 66, 1138 (1995).

[23] J. Pearl, Appl. Phys. Lett. 5, 65 (1964).

[24] We estimate $\lambda$ with the dirty-limit expression described in P. H. Kes and C. C. Tsuei, Phys. Rev. B 28, 5126 (1983). The coherence length $\xi(0)$ is estimated by $\xi(0)=$ $\sqrt{\Phi_{0} / 2 \pi H_{c 2}(0)}$ with the second critical field $H_{c 2}(0)$ at $T=0$.

[25] B. L. T. Plourde, D. J. Van Harlingen, N. Saha, R. Besseling, M. B. S. Hesselberth, and P. H. Kes, Phys. Rev. B 66, 054529 (2002).

[26] V. G. Kogan, V. V. Dobrovitski, J. R. Clem, Y. Mawatari and R. G. Mints, Phys. Rev. B 63, 144501 (2001).

[27] The rms flux noise for our system referred to the pick-up loop is $\sim 5 m \Phi_{0}$ per pixel in the images.

[28] F. Tafuri, J. R. Kirtley, P.G. Medaglia, P. Orgiani, and G. Balestrino, Phys. Rev. Lett. 92, 157006 (2004).

[29] T. Nishio, S. Okayasu, J. I. Suzuki, N. Kokubo, and K. Kadowaki. Phys. Rev. B 77, 052503 (2008).

[30] We note that the observed orientations are some of the metastable states stabilized by subtle uneven boundary confinement due to the edge roughness and/or possible weak bulk pinning in the disk. Otherwise, during the scanning, the orientation can be aligned along the scan direction of the pick-up loop due to the weak coupling between the loop and the vortices.

[31] Repeated field-cool measurements at the same magnetic fields show nearly identical vortex configurations, including the orientation of the patterns, when the vorticity is unchanged.

[32] We emphasize that the pinning site accommodates only one vortex and others are distributed around in the disk. This is distinct from phenomena observed in the micrometer-sized $\mathrm{Nb}$ mesas, where multi-fluxons are observed near the strong pinning site [21].

[33] The pinning site at the disk center can be found from a vortex pattern at $L=2$. 
2 